\title{
Improving the Security and Reliability of Information-Exchange in Telecommunication Systems with Mobile Objects

\author{
${ }^{\text {*1 }}$ Sergey P. Belov, ${ }^{2}$ Vladimir S, Serdyukov, ${ }^{3}$ Nikolay G. Zakharchenko, ${ }^{4}$ Natalia V Kolos, ${ }^{5}$ Sergey V. \\ Ponomarenko, ${ }^{6}$ Sergey A. Ponomarenko, ${ }^{7}$ Vitaly V. Vedritsky \\ $1,2,3,4,5,6,7$ Belgorod University of Cooperation, Economics and Law, Russia, 308023, Belgorod, Sadovaya st., \\ $116 \mathrm{a}$ \\ E-mail: belovssergei@rambler.ru,serdukov_vs@mail.ru,zng@bukep.ru,kolos.natalia1928@gmail.com,kaf- \\ otzi-spec@bukep.ru,www.major@yandex.ru,vedritskiy@mail.ru
}

Received: 07th February 2020, Accepted: 02nd March 2020, Published: 30th April 2020

\begin{abstract}
Based on the analysis of existing approaches to ensuring the security and reliability of information exchange in telecommunication systems with mobile objects, the article notes that the most promising approach to ensure the required performance of these features is the use of signal method. This method is based on the use of combined channel signals as information carriers, created on the basis of special codes and new spectrum spreading methods.

It is shown that a sufficiently large number of different classes of channel signals have been developed today, the use of which as information carrier in the telecommunication systems with mobile objects allows somehow solving the problem of ensuring a given level of security and reliability of information exchange in these systems. At the same time, it is too early to say that this task has already been fully resolved.

In particular, the results of time-frequency features obtained in the research process of a new class of combined signal-code constructions, created on the basis of special sets of radio pulses with linear frequency modulation as the basis designs, allow making a statement that their use as information carriers in telecommunication systems with mobile objects allows providing greater security and reliability of information exchange in these systems compared to the existing classes of combined channel signals, since the developed class of combined channel signals has both the property of invariance to Doppler frequency mismatch and the high level of noise immunity.
\end{abstract}

Keywords

Immunity, Telecommunication Systems, Doppler Effect.

\section{Introduction}

The current stage of social development is characterized by a continuous increase in remote interaction between subscribers and user requests for various types of multiservice services with the required quality, regardless of their location, which is currently mainly implemented on the basis of telecommunication systems with mobile objects, one of which is satellite telecommunication systems (STCS). The importance of these systems for the information transmission has significantly increased after the adoption of appropriate decisions on the development of the Arctic latitudes.

However, an intensive increase in the number of these systems, simultaneously transmitting information through the communication channels with limited time-frequency resources, leads to the appearance of interference noise. In addition, a significant decrease in the quality of multiservice services provided using the STCS is facilitated by the systems developed in recent years, designed to generate intentional noise in the communication channel or unauthorized access to the transmitted information.

Thus, it is necessary to ensure an appropriate level of noise immunity for the effective functioning of satellite telecommunication systems in a complex electronic environment.

Currently, various approaches have been created to ensure the security and reliability of information exchange in the STCS, among which it is customary to single out organizational, spatial, energy and signal methods in the qualification essence [1].

At the same time, an analytical review of a large number of references [1-22] made it possible to establish that the use of digital processing methods for channel signals in the modern STCS, allowing adaptively changing their time-frequency features, the development of new types of modulation and demodulation have led to the greatest use of signal methods in these systems to increase the security and reliability of information exchange. In this regard, the article considers the possibility of using a new class of combined signal-code structures obtained when using special sets of radio pulses with linear frequency modulation as the basis structures, with an aim of using them as information carriers in the STCS to increase the reliability and security of information exchange in these systems. 
Main Parts of Research

Material and Methods

The developed class of broadband noise-like signals is a combined signal, which is based on the use of a timefrequency matrix, each element of which is a linear frequency-modulated pulse with a different value of the frequency change rate (LFM-FWM signal). Such a class of signals can be written as follows in mathematical form:

$$
S(t)=\left\{\begin{array}{l}
S_{0} \cdot \sum_{l=1}^{L} \operatorname{rect}\left\{t-(l-1) \cdot \tau_{u}\right\} \cdot \exp \left(j \cdot\left(\omega_{0} \cdot\left(t-(l-1) \cdot \tau_{u}\right)+\left(N_{k}-N_{n}\right) \cdot \frac{\mu\left(t-(l-1) \cdot \tau_{u}\right)^{2}}{2}\right)\right) \\
\text { at } 0<t<L \cdot \tau_{u} \\
0 \text { at other } t
\end{array}\right.
$$

where: $\mathrm{S}_{0}$ - amplitude of signal envelope, hereinafter a constant value equal to $1 ; L$ - number of numerical sequence elements; $\omega_{0}$ - signal carrier frequency;

$N_{k}$ - number of number sequence from 1 to $L$;

$\mu$-steepness of the modulation feature of the LFM radio pulse (frequency change rate) associated with the frequency deviation $\Delta \mathrm{F}_{\mathrm{j}}$ and the duration

of number sequence element $\tau_{u}$ with a ratio $\mu=\frac{2 \cdot \pi \cdot \Delta F_{j}}{\tau_{u}}$;

$N_{n}$ - constant number, rect $(x)$ - rectangular "cutting" function defined by the expression:

$\operatorname{rect}\left\{t-(l-1) \cdot \tau_{u}\right\}=\left\{\begin{array}{l}1 \text { at }(\mathrm{k}-1) \cdot \tau_{u} \leq t \leq k \cdot \tau_{u}, \\ 0 \text { at }(\mathrm{k}-1) \cdot \tau_{u}>t>k \cdot \tau_{u} .\end{array}\right.$

In the future, we will assume that $N_{n}=(L+1) / 2$ leads to a symmetric arrangement of the LFM-FWM signal spectrum relative to the carrier frequency $\omega_{0}$. It should be noted that various options for the formation of numerical sequence $N_{k}$ and modulating functions as a whole are possible [1].

Figure 1 shows the structural diagram of one of the options for devices that allows formulating the proposed class of signals of FWM with LFM.

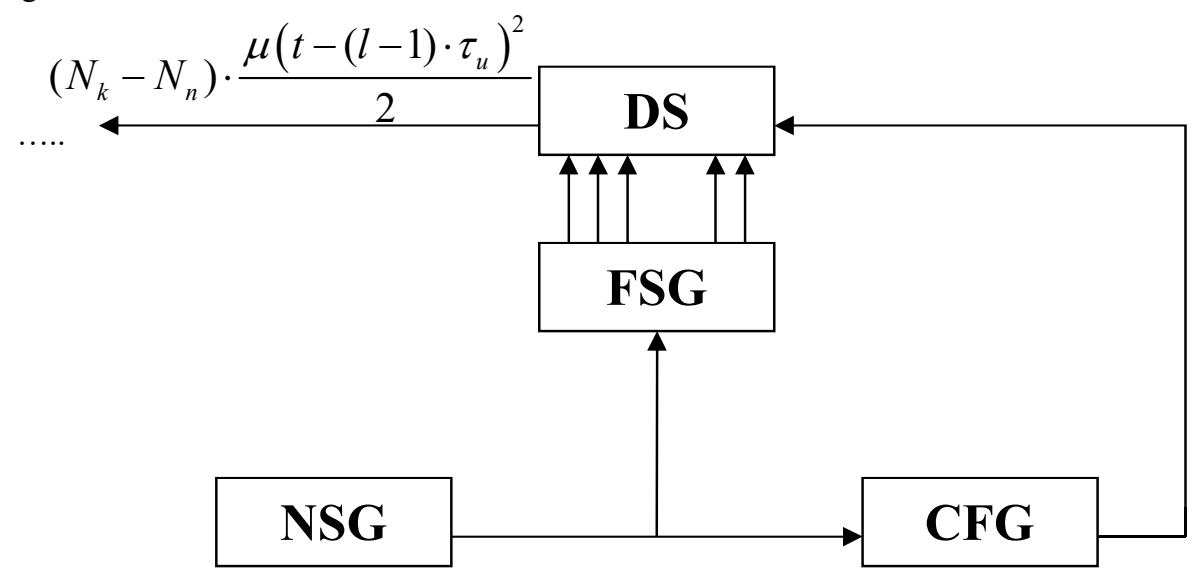

Figure 1: FWM with LFM Signal Generating Device

(where DS - Digital Switch, FSG - Frequency Spectrum Generator, CFG - Clock Frequency Generator, and NSG - Numerical Sequence Generator) 
The operation principle of the proposed scheme is as follows. The clock frequency generator (CFG) generates a signal with a clock frequency $\omega_{\tau_{u}}$ that determines the clock frequency of a numerical sequence generator (NSG) based on a linear feedback shift register, the state of which is characterized by a binary number determined by the state ( 1 or 0$)$ of each trigger included in the shift register, at each clock cycle.

As is known, there are $L=2^{n}-1$ states that provide all numbers $N_{k}$ from 1 to $L$ for a shift register that generates a maximum length sequence [1-3].

The frequency spectrum generator (FSG) generates a spectrum of linear frequency-modulated pulses with different slopes for each element of the frequency-time matrix.

$$
\begin{aligned}
& \omega_{0} \cdot\left(t-(l-1) \cdot \tau_{u}\right)+\left(N_{1}-N_{n}\right) \cdot \frac{\mu_{1}\left(t-(l-1) \cdot \tau_{u}\right)^{2}}{2}+\omega_{0} \cdot\left(t-(l-1) \cdot \tau_{u}\right)+\left(N_{2}-N_{n}\right) \cdot \frac{\mu_{2}\left(t-(l-1) \cdot \tau_{u}\right)^{2}}{2} \\
& +\ldots+\omega_{0} \cdot\left(t-(l-1) \cdot \tau_{u}\right)+\left(N_{k}-N_{n}\right) \cdot \frac{\mu_{k}\left(t-(l-1) \cdot \tau_{u}\right)^{2}}{2}
\end{aligned}
$$

All signals of the frequency spectrum are supplied to the corresponding first inputs of the digital switch (DS), the second input of which is supplied with a digital code from the NSG output. The digital switch associates each number $N_{k}$ with a predetermined signal from the frequency spectrum with linear frequency-modulated pulses $\omega_{0} \cdot\left(t-(l-1) \cdot \tau_{u}\right)+\left(N_{i}-N_{n}\right) \cdot \frac{\mu_{i}\left(t-(l-1) \cdot \tau_{u}\right)^{2}}{2}$ and only this signal is passed to the output.

The LFM-FWM signal thus obtained will have a duration equal to $T_{c}=L \cdot \tau_{u}$, and the width of the frequency band occupied by it can be determined from the following expression $\Delta F_{c}=(L-1) \cdot \Delta F_{j}$.

Hence, the base value of such a signal can be written in mathematical form as follows:

$B_{s}=L \cdot \tau_{u} \cdot(L-1) \cdot \Delta F_{j}$

The number of different element movements of the thus formed square matrix of $L$ size will be equal to:

$$
N=1 \cdot 2 \ldots L=L !
$$

When using the developed class of signals as information carrier in high-orbit STCS, it is very important to know the degree of their invariance to Doppler frequency mismatch, since this parameter largely determines the time-frequency costs of the indicated telecommunication systems for the search and synchronization of the signals used.

As is known [15], the uncertainty function is widely used as a quantitative estimate of invariance degree of a particular class of channel signals to Doppler frequency mismatch, which can be represented by the following formula in general form:

$$
\dot{\chi}_{i i}\left(\tau, F_{d}\right)=\frac{1}{2 E} \int_{-\infty}^{\infty} \dot{S}_{i}(t) \cdot \dot{S}_{i}^{*}(t-\tau) \cdot \exp \left(j \cdot 2 \pi \cdot F_{d} \cdot t\right) d t
$$

where: $\tau$ - time shift between signals, $F_{D}$ - Doppler frequency mismatch, E - signal energy, $\dot{S}_{i}(t)$ - envelope of the received $i$-th signal, $\dot{S}_{i}{ }^{*}(t-\tau)$ - complex conjugate envelope of $i$-th signal.

An analysis of the results of experimental studies of the cross sections of envelope LFM-FWM signals obtained at different values of the time shift between the signals $(\tau)$ and Doppler frequency mismatch $\left(F_{D}\right)$, a typical example of which is presented in Figure 2, allowed establishing that the developed classes of channel signals for a certain selection of frequency change rates for each of the LFM pulses, which are the time-frequency matrix 
elements, provide complete invariance to Doppler frequency mismatch $\left(F_{D}\right)$ within the limits of its real change (from 0 to $50 \mathrm{kHz}$ ).

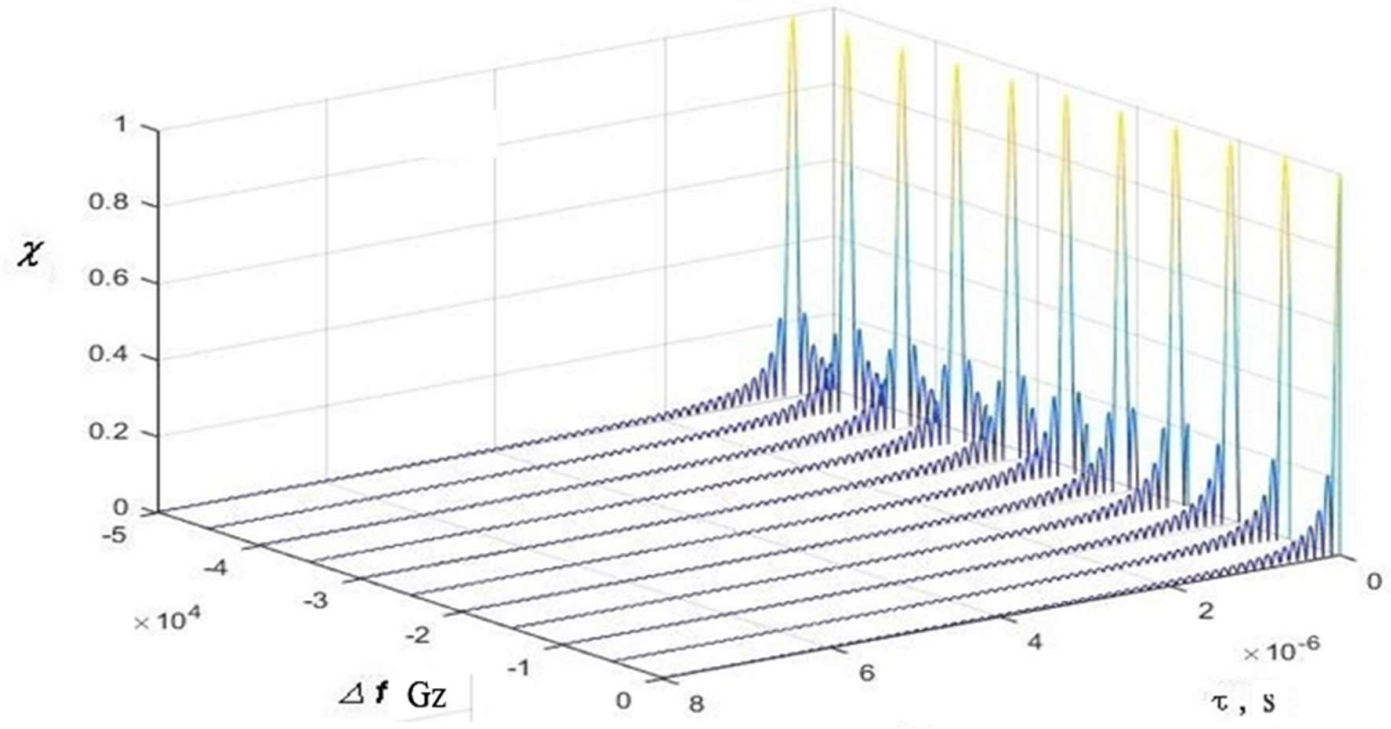

Figure 2: Values of LFM-FWM Signals

\section{Conclusions}

The presence of such a large volume of different LFM-FWM signals can significantly increase the noise immunity of satellite telecommunication systems for various types of attempts to unauthorized access to information transmitted by these systems.

It should also be noted that when implementing information exchange using high-orbit satellite telecommunication systems, the use of the developed class of LFM-FWM signals as information carriers in these systems, with a certain selection of the frequency change rate of each of the LFM pulses, which are the time-frequency matrix elements, will ensure complete invariance to Doppler frequency mismatch $\left(F_{D}\right)$ within its real change (from 0 to $50 \mathrm{kHz}$ ).

\section{Acknowledgements}

The studies were supported by the RFBR (Russian Foundation for Basic Research) grant No. 18-07-00356a

\section{References}

1. Tuzov, G.I. Statistical theory of reception of complex signals [Text] / - M.: Sov. Radio, 1977. - 400 P.

2. Noise-like signals in THE information transmission systems [Text] / V.B. Pestryakov, V.P. Afanasyev, V.L. Gurvich et al.: ed. by V.B. Pestryakov - M.: Sov. Radio, 1973. - 424 P.

3. Varakin, L.E. Theory of signal systems [Text]. - M.: Sov. Radio, 1978. - 304 P.

4. Volkov, L.N., Nemirovsky, M.S., Shinakov, Yu.S. Digital radio communication systems: basic methods and features [Text]: Study Guide. - M.: Eco Trends, 2005.

5. Galkin, V.A. Digital mobile radio communication [Text]. Textbook for universities. - M.: Goryachaya Liniya-Telekom, 2007. - $432 \mathrm{P}$.

6. Sklyar, Bernard. Digital communications. Theoretical foundations and practical application [Text]. 2nd edition, revised. Translated from English. - M.: Williams Publishing House, 2003 - 1104 p.

7. Garanin, M. B. Systems and networks for the information transmission [Text] / M. V. Garanin, V.I. Zhuravlev, S.V. Kunegin. - M.: Radio i svyaz, 2001. - 336 P.

8. Dickson, R.K. Broadband systems [Text]. Translated from English / Edited by V.I. Zhuravlev - M.: Svyaz, 1979. - $304 \mathrm{p}$.

9. Babin A.I. Radio frequency spectrum: efficiency of use and regulation offers, "Electrosvyaz" No. 7, 2009.

10. Mobile WiMAX - Part I: A Technical Overview and Performance Evaluation, August, 2006

11. Jeffrey G. Andrews, Ph.D. Fundamentals of WiMAX. Understand Broadband Wireless Networking. Prentice Hall, 2005.

12. Belov S.P., Zhylyakov E.G., Belov A.S. The possibility of using one class of complex signals with LFM for the transmission of voice data in the digital mobile communication systems. Questions of radio electronics. Ser. "Electronic Computer Facilities (ECF)." - M. - 2008. Edition 1. 161-171 P. 
13. Signal generation with linear frequency modulation [Text] / V.N. Kochemasov, L.A. Belov, V.S. Okoneshnikov - M.: Radio i svyaz, $1983-192$ p.

14. Gantmakher, V.E., Bystrov, N. E., Chebotarev, D. B. Noise-like signals. Analysis, synthesis, processing [Text]. - St. Petersburg: Nauka i technika, $2005-400$ p.

15. Cook C., Bernfeld M. Radar signals [Text]. M.: Sov. Radio. - 1971. - 568 p.

16. Borisov, V.I. Interference immunity of radio communication systems with the expansion of modulation signal spectrum by a carrier pseudorandom sequence / V.I. Borisov, V.M. Zinchuk, A.E. Limarev. - M. : Radio i svyaz, 2003. - 640 p.

17. Kuzovnikov, A.V. Study of construction methods for noise-immune communication systems using waveletmodulated signals / A.V. Kuzovnikov // Radio engineering and electronics. - M. - 2014, volume 59, No. 1. 67-77 P.

18. Chernousov A.V., Kuzovnikov A.V., Somov V.G., Principles of organizing an adaptive broadband communication system using wavelet-modulating functions. Telecommunication. 2014, No. 12: 14-17 P.

19. Belov S.P., Oleynik I.I., Rachinsky S.A. Comparative assessment of the frequency features of various classes of broadband channel signals. Optimal channel signals in digital transmission with frequency multiplexing // Scientific statements of BelSU. Ser. History. Politoology. Economics. Informatics. 2018 2(45): 394- 404 P.

20.Zhylyakov E.G., Belov S.P., Tuyakov S.V., Ursol D.V. On the best orthogonal basis for subband analysis and signal synthesis. Information systems and technologies. 2011. 2(64): 26-33 P.

21.Zhylyakov E.G., Belov S.P., Ushakov D.I., Starovoit I.A. The method of generating noise-resistant broadband signals. RF patent No. 2579759. 2016, Bull. 10.

22. S.P. Belov, S.A. Rachinsky, A.S. Belov, An.S. Belov, N.O. Efimov. On the effect of the Doppler frequency shift on the noise immunity of satellite telecommunication systems with complex signals // Scientific statements of BelSU. Ser. Economics. Informatics. 2017. - No. 9(258), issue 42. 179-187 P. 